\title{
Algorithm for analysis of administrative pediatric cancer hospitalization data according to indication for admission
}

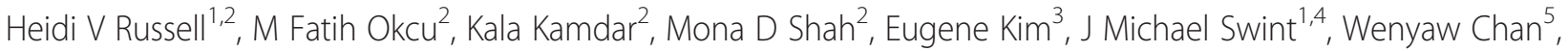
Xianglin $\mathrm{L} \mathrm{Du}^{6}$, Luisa Franzini ${ }^{1}$ and Vivian $\mathrm{Ho}^{7^{*}}$

\begin{abstract}
Background: Childhood cancer relies heavily on inpatient hospital services to deliver tumor-directed therapy and manage toxicities. Hospitalizations have increased over the past decade, though not uniformly across childhood cancer diagnoses. Analysis of the reasons for admission of children with cancer could enhance comparison of resource use between cancers, and allow clinical practice data to be interpreted more readily. Such comparisons using nationwide data sources are difficult because of numerous subdivisions in the International Classification of Diseases Clinical Modification (ICD-9) system and inherent complexities of treatments. This study aimed to develop a systematic approach to classifying cancer-related admissions in administrative data into categories that reflected clinical practice and predicted resource use.
\end{abstract}

Methods: We developed a multistep algorithm to stratify indications for childhood cancer admissions in the Kids Inpatient Databases from 2003, 2006 and 2009 into clinically meaningful categories. This algorithm assumed that primary discharge diagnoses of cancer or cytopenia were insufficient, and relied on procedure codes and secondary diagnoses in these scenarios. Clinical Classification Software developed by the Healthcare Cost and Utilization Project was first used to sort thousands of ICD-9 codes into 5 mutually exclusive diagnosis categories and 3 mutually exclusive procedure categories, and validation was performed by comparison with the ICD-9 codes in the final admission indication. Mean cost, length of stay, and costs per day were compared between categories of indication for admission.

Results: A cohort of 202,995 cancer-related admissions was grouped into four categories of indication for admission: chemotherapy ( $N=77,791,38 \%)$, to undergo a procedure $(N=30,858,15 \%)$, treatment for infection $(N=30,380,15 \%)$, or treatment for other toxicities $(\mathrm{N}=43,408,21.4 \%)$. The positive predictive value for the algorithm was $>95 \%$ for each category. Admissions for procedures had higher mean hospital costs, longer hospital stays, and higher costs per day compared with other admission reasons $(p<0.001)$.

Conclusions: This is the first description of a method for grouping indications for childhood cancer admission within an administrative dataset into clinically relevant categories. This algorithm provides a framework for more detailed analyses of pediatric hospitalization data by cancer type.

Keywords: Cancer, Health administrative data, Healthcare utilization, Child

\footnotetext{
* Correspondence: vho@rice.edu

${ }^{7}$ Baker Institute for Public Policy, Rice University, MS 40, P.O. Box 1892,

Houston, TX 77251-1892, USA

Full list of author information is available at the end of the article
} 


\section{Background}

Approximately 18,000 children under 19 years old are diagnosed with a childhood cancer annually in the United States [1]. One quarter of these children have leukemia, and other common diagnoses include lymphomas, central nervous system tumors, sarcomas, and neuroblastoma. Dramatic improvements in survival over the past few decades have resulted from use of complex multimodal therapies, often associated with high risks of side effects. These medically intense treatments rely heavily on inpatient hospital resources. As the focus on healthcare utilization increases, understanding the resources required to treat children with cancer becomes more critical.

Inpatient hospital needs for childhood cancer have grown at different rates by diagnoses. Price et al. identified admissions of children with cancer as the primary discharge diagnosis and compared the mean costs in 2000 and 2009. In 2009, the overall mean cost per cancerrelated admission was $\$ 40,400$ and had increased by 36\% from 2000 after accounting for inflation. The mean cost per Hodgkin lymphoma admission increased by $12 \%$ to $\$ 28,400$, central nervous system tumor admissions increased by $61 \%$ to $\$ 39,400$, and leukemia admissions by $32.3 \%$ to $\$ 55,700$ during this same period [2]. Berry et al. described a $3 \%$ decrease in the prevalence of admission of children with a diagnosis of bone malignancies from 2004 to 2009, while the prevalence of admission of children with a diagnosis of acute non-lymphoid leukemia increased $10.6 \%$ [3]. Changes in the incidence of new cancer diagnoses are insufficient to explain these differences by disease [1], suggesting that changes in clinical practice play a major role. However, the complex nature of multiple cancer diagnoses, treatments, and toxicities, combined with limited research resources, means that some prioritization for studying clinical practices is required. Therefore, we wanted to identify changes in the patterns of childhood cancer admissions at a national level that may account for the differences in hospital costs and prevalence with the aim of identifying areas with the greatest increases for future studies.

The current evidence for increased use of inpatient resources is difficult to relate to oncology practice. Both Price et al. and Berry et al. used cancer diagnoses to distinguish between admissions. However, childhood cancer is a chronic condition and not, in itself, a reason for hospital admission. Most admissions for cancer are for scheduled treatment (e.g., to receive chemotherapy) or are unplanned for management of toxicities arising from cancer or its treatment [4-7]. Anticipated treatment regimens are often determined at a national cooperative group level and directly influence the frequency and severity of toxicities $[8,9]$. The reason for admission is a major determinant of the type and amount of resources used [10,11]. For example, patients admitted for chemotherapy will usually receive their pre-determined chemotherapy and be discharged quickly, whereas patients admitted for treatment of an infection are at higher risk of needing intensive care services [12].

Numerous prospective childhood cancer clinical trials have described treatment requirements and toxicities. This type of research has limited application to the study of childhood cancer hospitalizations as a whole $[13,14]$ because it only captures patients and events associated with individual trials. Less than half of children enroll on clinical trials when first diagnosed with cancer $[15,16]$ and this subset may not be wholly representative of the childhood cancer population. Adolescents have historically been underrepresented on clinical trials $[15,17]$ and children with pre-existing medical conditions are less likely to be eligible for participation [16]. Clinical trials must be available for the patient's cancer, a limiting factor for patients with rare tumors or those receiving care at institutions not participating in childhood cancer clinical trials [15]. Furthermore, focusing on clinical trials will fail to capture palliative or end-of-life care, an important and costly part of medical services for childhood cancer [18]. Administrative data, or data passively collected for other indications, provides access to a more diverse population of patients and may be more accurate for studying admission patterns on a national level [19]. The Kids Inpatient Database (KID), compiled by the Healthcare Quality and Utilization Project (HCUP) and sponsored by the Agency for Healthcare Research and Quality, is a nationally representative database that samples approximately $80 \%$ of pediatric discharges from community hospitals in the United States. A version is produced every 3 years and contains between 2.5 and 3.4 million de-identified admission records from 4,839 to 5,128 hospitals in 36 to 44 states [20]. All administrative datasets pose challenges, including the structure and quality of the data and generalizability [21]. KID uses hospital admission as the primary unit of data; patients are de-identified and repeat admissions cannot be accounted for, and diagnoses and procedures are defined by billing codes. However, these limitations are balanced by the possibility of generating national hospitalization estimates.

Administrative datasets that rely on billing codes pose two challenges for studying complex diseases. The first is the assignment of the primary discharge diagnosis by coders remote from the clinical treatment team [21]. The second is the quantity of possible individual codes. The 9th version of the International Statistical Classification of Diseases and Related Health Problems (ICD-9) coding system includes approximately 12,000 diagnostic and 3,500 procedure codes. Furthermore, codes change over time. To facilitate analysis using ICD codes, HCUP developed Clinical Classification Software (CCS) which 
groups the thousands of ICD-9 codes into 260 mutually exclusive diagnostic groups and 231 mutually exclusive procedure groups [22]. Most of the CCS groups represent fairly homogenous diagnoses or procedures, but others combine conditions or procedures within a body system [22]. CCS groups were developed for use across a broad spectrum of potential diagnoses and may not reflect a particular clinical situation such as childhood cancer [23].

To study childhood cancer admission patterns and trends on a national level, we first sought to develop a systematic approach to classifying cancer-related admissions from administrative data into categories that reflected clinical practice. The following report describes a multistep process for stratifying the indication for hospital admission into four categories: chemotherapy, to undergo a procedure, treatment for infection, or treatment for other toxicities. To overcome the challenges of sorting thousands of potential ICD-9 diagnostic and procedure codes, the 491 CCS groups were incorporated into the algorithm as an initial step in its development. To validate the accuracy of the CCS in correctly allowing admissions to be sorted into the four indications for admission, the ICD-9 codes in each category were individually reviewed after assignment. Finally, to test our assumption that utilization would be related to the indication for admission, mean estimates of utilization measures and rates of high intensity events were compared between admission indication categories.

\section{Methods}

\section{Data source}

A cohort of cancer-related admissions was identified from the full KID dataset for the years 2003, 2006, and 2009 (Figure 1A). Each KID admission contained up to 15 discharge diagnoses in 2003 and 2006, and 25 discharge diagnoses in 2009. Cancer-related admissions were defined as any admission with a cancer diagnosis (CCS diagnostic groups 11 to 43) in any of the discharge diagnoses [2]. All other admissions were excluded from further analysis. In addition to diagnostic codes, each admission contained up to 15 procedural codes, basic demographic information on the patient (i.e., age, sex, healthcare payer), status of the patient upon discharge (i.e., alive or dead), and hospital location by state. States participating in HCUP elect to submit patient race/ ethnicity data and the hospital day on which procedures occurred. Utilization data on each admission included length of stay (LOS) and total admission charge. This deidentified dataset was considered exempt from human subjects review by the institutional review board of the Houston branch of the University of Texas Health Science Center. The dataset was accessed after completion of a data use agreement with HCUP.

\section{Admission indication definitions and algorithm development}

Definitions of clinically meaningful diagnostic categories, procedure categories, and admission indications were developed by consensus by a group of four pediatric oncologists (HR, FO, KK, MS) and a pediatric oncology surgeon (EK), all but one with experience in clinical processes through formal professional development. In addition, two members held clinical management roles, two with advanced training in epidemiology and one with advanced training in health economics/health services research.

Clinical experience and relevant literature supported four distinct indications for hospitalization of a child with cancer: to deliver chemotherapy (Intent-Chemotherapy), to perform a procedure (Intent-Procedure), to treat an infection (Intent-Infection), or to manage a non-infectious toxicity (Intent-Toxicity). Our approach to categorizing admissions into these four indications required two main assumptions. First, we assumed that a discharge diagnosis of cancer or cytopenia (i.e., neutropenia, anemia, thrombocytopenia) did not provide a sufficient reason for admission: cancers are chronic conditions and cytopenias are managed in an outpatient setting in the absence of other complications. Second, we assumed that when the primary discharge diagnosis did not sufficiently describe the reason for admission, the secondary discharge diagnosis would be an adequate substitute [24].

We defined an indication for each admission according to a stepwise mutually exclusive algorithm (Figure 1B). The first two indications, Intent-Chemotherapy and IntentProcedure, were identified by the performance of chemotherapy or a cancer-related procedure, respectively, during the first 2 days of hospitalization. Intent-Chemotherapy was identified first so that admissions that included minor procedures performed prior to, but not delaying, chemotherapy would be considered chemotherapy admissions rather than procedure admissions. The third and fourth indications, Intent-Infection and Intent-Toxicity, were identified from the remaining admissions by the presence of an infection or non-infectious toxicity code, respectively, as the primary discharge diagnosis or the secondary discharge diagnosis if the primary discharge diagnosis was a cancer or cytopenia. Intent-Infection admissions were identified before Intent-Toxicity to allow admissions with a primary diagnosis of cytopenia and a secondary diagnosis of infection to be classified as an Intent-Infection admission.

\section{CCS categories and validation}

To implement the above algorithm, each primary and secondary discharge diagnostic code and each procedure code needed to be categorized into five diagnostic categories (malignancy, chemotherapy, infection, non-infectious toxicities, and other) and three procedure categories 


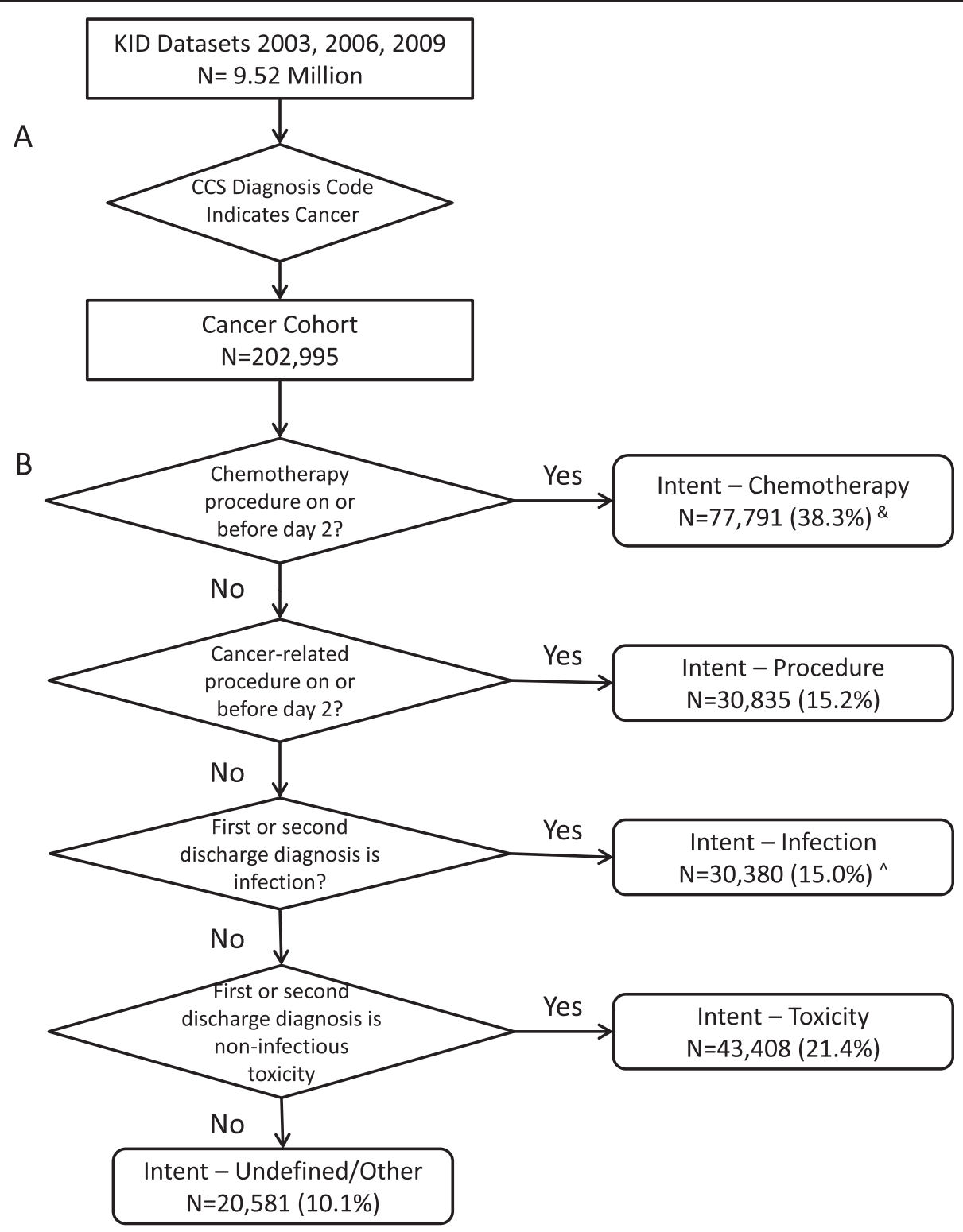

Figure 1 Multi-step algorithm for stratifying childhood-cancer admissions. (A) Identification of a cohort of cancer-related admissions from the full KID dataset. (B) Stratification of admissions into indications for admissions. KID indicates Kids Inpatient Database; CCS indicates Clinical Classification Software. \&: Includes admissions with chemotherapy as primary procedure from states with no reported procedure dates. $\wedge$ : Excludes CCS diagnostic group 237 non-infectious ICD-9 codes and non-cytopenia toxicities.

(chemotherapy, cancer procedures, and other). Chemotherapy is defined as both a procedure and diagnosis within the CCS and ICD-9 systems. This categorization was performed using the 491 CCS groups after examination by the physician group (HR, FO, KK, MS, EK) of the definition of each CCS group and the ICD-9 codes it contained. The complete categorization is presented in Additional file 1: Table S1. CCS diagnostic groups 11 through 43 were categorized as malignancy and CCS group 45 was categorized as chemotherapy. CCS diagnostic groups were categorized as infections if they included definitive or presumed infections by any microbe, fever, and/or shock. CCS diagnostic groups were categorized as non-infectious toxicities if they included toxicities arising from the cancer pathology or treatment for the cancer not previously categorized as infection, and were further sub-classified into cytopenias (CCS diagnostic groups 59, 60, and 62-64) and non-cytopenic toxicities (all others). CCS diagnostic group 237 ("Complication of device, implant or graft") was subdivided into infections (ICD-9 999.31, 996.62, 996.67, 996.69) and noninfections (all others) as it contained large proportions of both. Diagnostic groups not related to the malignancy were categorized as "other". Such diagnoses included 
pregnancy/delivery, congenital disorders, asthma, other established pediatric diagnoses, mental/behavioral disorders, and trauma. Chemotherapy procedures were limited to CCS procedure group 224. Other CCS procedure groups were categorized as cancer-related procedures if they included any procedure for the diagnosis or treatment of the malignancy or management of a treatment-related toxicity. Imaging studies and blood product transfusions were excluded. See Additional file 2 for a do-file of the CCS classification and algorithm.

To validate the accuracy of the CCS in correctly sorting admissions into admission indication categories, the ICD-9 codes for the primary and secondary discharge diagnoses and procedures occurring in the first 2 days of hospitalization were reviewed after admissions were assigned. Review of the individual codes was limited to the $90 \%$ most frequent ICD-9 codes or codes appearing more than once within that admission indication, whichever limit was achieved first. The initial sorting and review of ICD-9 codes was performed by a pediatric oncologist (HR). The clinical team then reviewed the categorization and any conflicts were resolved by discussion until consensus was obtained. The positive predictive value (PPV) of each admission indication as sorted by CCS groups was determined by calculating the percentage of the true positives (i.e., admissions with ICD-9 codes appropriate for the admission indication) within each admission category. Sensitivity was further determined by identifying admissions mis-classified by CCS groupings. We desired the algorithm to have a PPV of $\geq 95 \%$. Because admission numbers were large, 95\% confidence intervals (CI) were determined using simple asymptotic methods [25]. All analysis was performed on STATA version 11 (Stata Corp., College Station, TX, USA).

\section{Comparison of admission indications}

We compared the admission indications by frequency of resource-intense events and utilization measures using descriptive statistics and analysis of variance. Admissions associated with hematopoietic stem cell transplantation (HCT) or requiring intensive care unit (ICU) support are resource intense events occurring frequently in childhood cancer treatment [10,12,26,27]. Admissions associated with HCT were defined as those including a CCS procedure code 64 (ICD-9 41.xx). Admissions associated with ICU services were defined as those with diagnostic codes of respiratory arrest (CCS 131), cardiac arrest (CCS 107) or shock (CCS 249). Total admission costs were estimated by converting charges to costs using cost-to-charge ratios [28]. A mean cost per day for each admission was calculated as the quotient of total cost of admission over LOS. All costs were inflated to 2009 US\$ using the All-Urban Consumer Price Index [29]. A $p$-value $<0.05$ was considered significant.

\section{Results}

Overall cancer subset

We identified 202,995 admissions with a cancer diagnosis from the 2003, 2006 and 2009 KIDs. Characteristics of these admissions are presented in Table 1.

\section{Admission indication}

The cohort of childhood cancer admissions (Figure 1A) was sorted into admission indications using CCS diagnostic and procedure categories (Figure 1B). Review of the ICD-9 codes of the admissions in each indication validated the accuracy of this sorting process. The distribution of admissions by indication is presented in Table 2 .

Intent-Chemotherapy admissions were identified first from the entire cancer cohort. The initial definition of

Table 1 Characteristics of childhood cancer admissions

\begin{tabular}{|c|c|}
\hline & $\begin{array}{c}\mathrm{N}=202,995 \\
\mathrm{n}(\%)\end{array}$ \\
\hline \multicolumn{2}{|l|}{ Year } \\
\hline 2003 & $62,783(30.9 \%)$ \\
\hline 2006 & $66,397(32.7 \%)$ \\
\hline 2009 & $73,815(36.4 \%)$ \\
\hline \multicolumn{2}{|l|}{ Age at admission } \\
\hline$<1 \mathrm{y}$ & $6,722(3.3 \%)$ \\
\hline 1 to $4 y$ & $48,461(23.9 \%)$ \\
\hline 5 to $9 y$ & 43,523 (21.4\%) \\
\hline 10 to $14 \mathrm{y}$ & $42,801(21.1 \%)$ \\
\hline$>15 y$ & $60,982(30.0 \%)$ \\
\hline \multicolumn{2}{|l|}{ Gender } \\
\hline Male & $112,323(55.3 \%)$ \\
\hline Female & $90,672(44.67 \%)$ \\
\hline \multicolumn{2}{|l|}{ Payer^ } \\
\hline Public\& & $73,973(36.4 \%)$ \\
\hline Private & $113,027(55.7 \%)$ \\
\hline Other & $15,678(7.7 \%)$ \\
\hline \multicolumn{2}{|l|}{ Race $\wedge$} \\
\hline White & $93,681(46.2 \%)$ \\
\hline Black & $17,571(8.7 \%)$ \\
\hline Hispanic & $37,877(18.8 \%)$ \\
\hline Other (Asian, Native American, "other") & $14,782(7.3 \%)$ \\
\hline \multicolumn{2}{|l|}{ CCS category of primary diagnosis } \\
\hline Malignancy & $47,853(23.6 \%)$ \\
\hline Chemotherapy & $74,955(36.9 \%)$ \\
\hline Infection & $30,169(14.9 \%)$ \\
\hline Non-Infectious toxicity & $40,725(20.1 \%)$ \\
\hline Other & $9,293(4.6 \%)$ \\
\hline
\end{tabular}

\&Represents both Medicaid and Medicare; ^Percentages do not sum to 100 owing to missing data. CCS indicates Clinical Classification Software. 
Table 2 Positive predictive values of cancer-related admissions categorized by indication for admission

\begin{tabular}{|c|c|c|c|c|c|c|c|c|c|}
\hline \multirow[b]{2}{*}{ Admission indication } & \multicolumn{5}{|c|}{ CCS category of primary diagnosis } & \multicolumn{3}{|c|}{ ICD-9 code } & \multirow[b]{2}{*}{$\begin{array}{c}\text { Positive predictive } \\
\text { value (95\% confidence } \\
\text { interval) }\end{array}$} \\
\hline & $\begin{array}{c}\text { Malignancy } \\
N=47,853 \\
n(\%)\end{array}$ & $\begin{array}{c}\text { Chemotherapy toxicity } \\
\mathrm{N}=74,955 \\
\mathrm{n}(\%)\end{array}$ & $\begin{array}{c}\text { Infection } \\
N=30,169 \\
n(\%)\end{array}$ & $\begin{array}{c}\text { Non-infectious toxicity } \\
\mathrm{N}=40,725 \\
\mathrm{n}(\%)\end{array}$ & $\begin{array}{c}\text { Other } \\
N=9,293 \\
\text { n (\%) }\end{array}$ & Reviewed & True + & False + & \\
\hline $\begin{array}{l}\text { Intent-chemotherapy } \\
\qquad N=77,791\end{array}$ & $6,985(14.6 \%)$ & $67,330(89.8 \%)$ & $1,428(4.7 \%)$ & $1,824(4.5 \%)$ & $224(2.4 \%)$ & $54,125 \#$ & 53,932 & 193 & $99.6 \%(99.6-99.7 \%)$ \\
\hline $\begin{array}{l}\text { Intent-procedure } \\
\qquad N=30,835\end{array}$ & $20,188(42.2 \%)$ & $1,585(2.1 \%)$ & $3,968(13.2 \%)$ & $3,258(8.0 \%)$ & $1,836(19.8 \%)$ & $45,855^{\wedge}$ & 45,249 & 606 & $98.7 \%(98.6-98.8 \%)$ \\
\hline $\begin{array}{l}\text { Intent-infection } \\
\qquad N=30,380\end{array}$ & $3,017(6.3 \%)$ & $249(<1 \%)$ & 23,468 (77.8\%) & $3,646(9.0 \%)$ & - & 28,887 & 28,255 & 632 & $97.8 \%(98.6-97.9 \%)$ \\
\hline $\begin{array}{l}\text { Non-infection toxicities } \\
\qquad N=43,408\end{array}$ & $9,607(20.1 \%)$ & $499(<1 \%)$ & $1,305(4.3 \%)$ & $31,997(78.6 \%)$ & - & 39,485 & 38,565 & 920 & $97.7 \%(97.5-97.8 \%)$ \\
\hline $\begin{array}{l}\text { Undefined } \\
\qquad N=20,581\end{array}$ & $8,056(16.8 \%)$ & $5,292(7.1 \%)$ & - & - & $7,233(77.8 \%)$ & & & & \\
\hline
\end{tabular}

\#Admissions associated with a date of chemotherapy delivery.

$\wedge$ Procedures. 
Intent-Chemotherapy admissions identified 69,922 admissions with chemotherapy delivered in the first 2 days of hospitalization. However, only $79 \%$ of admissions with a primary CCS diagnosis of chemotherapy were included, an unexpectedly low proportion. Several states did not report dates of procedures, resulting in 10,562 (14.1\%) admissions in this group without a date of chemotherapy delivery. Therefore, the Intent-Chemotherapy definition was modified to include admissions with chemotherapy delivery as the CCS group of the primary procedure, which added 7,869 admissions to Intent-Chemotherapy. Of the IntentChemotherapy admissions with an identifiable date of chemotherapy administration, $77.4 \%(\mathrm{~N}=54,125)$ received chemotherapy on Day 0 of hospitalization, $16.2 \%(\mathrm{~N}=$ $11,315)$ on Day 1 and $5.61 \%(\mathrm{~N}=3,923)$ on Day 2. In a few admissions $(\mathrm{N}=193,<1 \%)$ the date of chemotherapy was recorded as negative and these were considered false positives. Chemotherapy procedure codes were included in an additional 10,583 admissions not classified as Intent-Chemotherapy. In these admissions, the median first day of chemotherapy was hospital Day 5 (range Day 3-172).

Intent-Procedure admissions were identified from the remaining cancer cohort. Both ICD-9 procedure codes and primary ICD-9 diagnostic codes (Table 2) from admissions in this indication category were reviewed. A total of 50,225 procedures were performed in the first 2 days of the 30,835 admissions identified (average 1.4 procedures per admission, range $1-14$ ). The 10 most frequent procedures associated with these admissions are listed in Table 3. Of the 606 (1.3\%) procedures not associated with

Table 3 Most common ICD-9 procedure codes in the first 2 hospital days of Intent-Procedure admissions

\begin{tabular}{|c|c|c|}
\hline $\begin{array}{l}\text { ICD-9 } \\
\text { procedure } \\
\text { code }\end{array}$ & $\begin{array}{l}N=50,225 \wedge \\
n(\%)\end{array}$ & Description \\
\hline 41.31 & $5,185(10.3 \%)$ & Biopsy of bone marrow \\
\hline 38.93 & 4,066 (8.1\%) & $\begin{array}{l}\text { Venous catheterization, not elsewhere } \\
\text { classified }\end{array}$ \\
\hline 01.59 & $3,160(6.3 \%)$ & $\begin{array}{l}\text { Other excision or destruction of lesion } \\
\text { or tissue of brain }\end{array}$ \\
\hline 03.31 & $2,705(5.4 \%)$ & Spinal tap \\
\hline 86.07 & $2,264(4.5 \%)$ & $\begin{array}{l}\text { Insertion of totally implantable vascular } \\
\text { access device [VAD] }\end{array}$ \\
\hline 85.05 & 1,147 (2.3\%) & $\begin{array}{l}\text { Incision with removal of foreign body or } \\
\text { device from skin and subcutaneous tissue }\end{array}$ \\
\hline 02.2 & $1,076(2.1 \%)$ & Ventriculostomy \\
\hline 40.11 & $1,038(2.1 \%)$ & Biopsy of lymphatic structure \\
\hline 40.3 & $827(1.7 \%)$ & Regional lymph node excision \\
\hline 54.4 & $819(1.6 \%)$ & $\begin{array}{l}\text { Excision or destruction of peritoneal } \\
\text { tissue }\end{array}$ \\
\hline
\end{tabular}

$\wedge 50,225$ procedures representing 1,036 individual ICD-9 procedure codes. cancer or treatment, 571 were appendectomy (47.xx) and 35 were umbilical vein catheterization (38.92).

Intent-Infection admissions were then identified from the remaining cancer cohort and ICD-9 codes of all primary and secondary diagnoses reviewed. Cytopenias, particularly neutropenia, were the primary diagnostic codes of $12 \%$ of the Intent-Infection admissions, and malignancy accounted for another 10\% (Table 2). The most frequent primary and secondary ICD-9 codes attributed to CCS groups categorized as infections are presented in Table 4. The most frequent secondary ICD-9 codes for these admissions varied slightly from those of the primary infection diagnosis (Table 4). Codes for fever, device infection, pneumonia, bacteremia, and upper respiratory tract infections were common regardless of whether the infection was the primary or secondary diagnosis. Codes listed only in primary infection diagnoses (i.e., septicemia and influenza) were the 11th and 20th (respectively) secondary diagnoses. Conversely, urinary tract infections and C. difficile infections were the 11th and 13th most common primary diagnoses. A notable exception was candidiasis of the mouth: despite it being the 5 th most common secondary infection diagnosis, it was the 90th most common primary infection diagnosis. ICD-9 codes for infections were associated with 659 Intent-Toxicity admissions and 91 Undefined/Other admissions resulting in a sensitivity of $97.4 \%$ (95\% CI, 97.2-97.6\%).

The final admission indication to be identified was Intent-Toxicity. Again, ICD-9 codes of primary and secondary diagnoses were reviewed. The most common first and second ICD-9 codes in Intent-Toxicity (Table 5) demonstrated that cytopenias remained a common diagnosis even after removing those admissions with an infection as the secondary diagnosis. Of the $51 \%$ of admissions in this indication with a primary diagnosis code describing a cytopenia, the secondary diagnosis was malignancy in $79 \%$ or a second cytopenia in $15.8 \%$. Non-infectious toxicity ICD-9 codes were associated with 491 Intent-Infection admissions and 510 Undefined/Other admissions, resulting in a sensitivity of $97.5 \%$ (95\% CI, 97.3-97.6\%).

After accounting for the four predefined indications for admission, 20,581 admissions (10.1\%) remained unclassified. Only $35 \%$ of these had a primary diagnosis with a CCS category of "other". ICD-9 V578.9 (Care involving other specified rehabilitation procedures) was the most frequent code and was associated with 509 admissions. Five of the 10 most frequent diagnostic codes were maternal/ fetal in nature (ICD-9 648.91, 664.01, V300.0, 659.71, and 645.11). Almost $17 \%$ of admissions with malignancy and $7 \%$ of admissions with chemotherapy as the CCS category of the primary diagnosis remained unclassified by this algorithm. For admissions with malignancy as a primary code, malignancy was the second diagnostic code in $38.7 \%$ and "other" in $61.1 \%$. 
Table 4 Most common ICD-9 diagnosis codes observed in Intent-Infection admissions

\begin{tabular}{|c|c|c|c|c|c|}
\hline \multicolumn{6}{|c|}{ Intent-Infection } \\
\hline \multicolumn{3}{|c|}{ CCS category of primary diagnosis of infection $\wedge$} & \multicolumn{3}{|c|}{$\begin{array}{l}\text { CCS category of primary diagnosis of malignancy or non-infectious toxicity } \\
\text { Secondary CCS category of secondary diagnosis of infection }{ }^{\&}\end{array}$} \\
\hline $\begin{array}{l}\text { ICD-9 } \\
\text { diagnosis } \\
\text { code }\end{array}$ & $\begin{array}{l}\mathrm{N}=23,468 \\
\mathrm{n}(\%)\end{array}$ & Description & $\begin{array}{l}\text { ICD-9 } \\
\text { diagnosis } \\
\text { code }\end{array}$ & $\begin{array}{l}N=6,912 \\
n(\%)\end{array}$ & Description \\
\hline 780.6 & $3,316(14.6 \%)$ & $\begin{array}{l}\text { Fever and other physiologic disturbances } \\
\text { of temperature regulation }\end{array}$ & 780.61 & $951(13.8 \%)$ & Fever \\
\hline 996.62 & $2,586(11 \%)$ & $\begin{array}{l}\text { Infection and inflammatory reaction due } \\
\text { to other vascular device, implant, and graft }\end{array}$ & 780.6 & $903(13.1 \%)$ & $\begin{array}{l}\text { Fever and other physiologic disturbances } \\
\text { of temperature regulation }\end{array}$ \\
\hline 486 & $1,671(7.2 \%)$ & Pneumonia, organism unspecified & 790.7 & $585(8.5 \%)$ & Bacteremia \\
\hline 780.60 & $1,522(6.5 \%)$ & Fever, unspecified & 486 & $506(7.4 \%)$ & Pneumonia, organism unspecified \\
\hline 790.7 & 1,392 (5.9\%) & Bacteremia & 112.0 & $291(4.2 \%)$ & Candidiasis of mouth \\
\hline 999.31 & $907(3.9 \%)$ & $\begin{array}{l}\text { Infection due to central venous } \\
\text { catheter }\end{array}$ & 996.62 & $285(4.1 \%)$ & $\begin{array}{l}\text { Infection and inflammatory reaction due } \\
\text { to other vascular device, implant, and graft }\end{array}$ \\
\hline 038.9 & $744(3.2 \%)$ & Unspecified septicemia & 599.0 & $279(4.1 \%)$ & Urinary tract infection, site not specified \\
\hline 465.9 & $710(3.0 \%)$ & $\begin{array}{l}\text { Acute upper respiratory infections } \\
\text { of unspecified site }\end{array}$ & 008.45 & $233(3.4 \%)$ & Intestinal infection due to Clostridium difficile \\
\hline 079.99 & $638(2.7 \%)$ & Unspecified viral infection & 780.60 & $208(3.0 \%)$ & Fever, unspecified \\
\hline 487.1 & $613(2.6 \%)$ & $\begin{array}{l}\text { Influenza with other respiratory } \\
\text { manifestations }\end{array}$ & 465.9 & $156(2.3 \%)$ & $\begin{array}{l}\text { Acute upper respiratory infections of } \\
\text { unspecified site }\end{array}$ \\
\hline
\end{tabular}

Ancludes 475 individual ICD-9 diagnosis codes.

\& Includes 322 individual ICD-9 diagnosis codes.

\section{Comparison of utilization by admission indication}

Mean resource utilization, and frequency of HCT, ICU use, and death were compared across admission indications (Figure 2). Significant differences $(p<0.001)$ between measures of utilization occurred in each comparison. Intent-Procedure admissions were associated with higher mean costs of hospitalization, longer hospital stays, and higher costs per day compared with other admission indications. Rates of ICU use and death were significantly lower in Intent-Chemotherapy than in other indications. The frequency of HCT procedures was highest in IntentChemotherapy admissions and Intent-Toxicity admissions.

Table 5 Most common ICD-9 diagnosis codes observed in Intent-Toxicity admissions

\begin{tabular}{|c|c|c|c|c|c|}
\hline \multicolumn{6}{|c|}{ Intent-Toxicity } \\
\hline \multicolumn{3}{|c|}{ CCS category of primary diagnosis of toxicity ${ }^{8 \wedge}$} & \multicolumn{3}{|c|}{$\begin{array}{l}\text { CCS category of primary diagnosis of malignancy or chemotherapy } \\
\text { CCS category of secondary diagnosis of non-infection toxicity\# }\end{array}$} \\
\hline $\begin{array}{l}\text { ICD-9 diagnosis } \\
\text { code }\end{array}$ & $\begin{array}{c}N=33,302 \\
n(\%)\end{array}$ & Description & $\begin{array}{l}\text { ICD-9 diagnosis } \\
\text { code }\end{array}$ & $\begin{array}{l}N=10,106 \\
n(\%)\end{array}$ & Description \\
\hline 288.0 & $8,109(24.4 \%)$ & Neutropenia & 284.8 & $806(8.0 \%)$ & Other specified aplastic anemias \\
\hline 288.00 & $5,351(16.1 \%)$ & Neutropenia NOS & 331.4 & $734(7.3 \%)$ & Obstructive hydrocephalus \\
\hline 284.8 & $1,920(5.8 \%)$ & Other specified aplastic anemias & 288.0 & $706(7.0 \%)$ & Neutropenia \\
\hline 288.03 & $1,349(4.1 \%)$ & Drug induced neutropenia & 780.39 & $507(5.0 \%)$ & Other convulsions \\
\hline 276.51 & $1,195(3.6 \%)$ & Dehydration & 287.5 & 437 (4.3\%) & Thrombocytopenia, unspecified \\
\hline 276.5 & $658(2.0 \%)$ & Volume depletion & 284.1 & $330(3.3 \%)$ & Pancytopenia \\
\hline 780.39 & $616(1.9 \%)$ & Other convulsions & 288.00 & $232(2.3 \%)$ & Neutropenia NOS \\
\hline 996.85 & $433(1.2 \%)$ & $\begin{array}{l}\text { Complications of transplanted } \\
\text { bone marrow }\end{array}$ & 276.1 & $200(2.0 \%)$ & Hyposmolality and/or hyponatremia \\
\hline 787.01 & $393(1.2 \%)$ & Nausea with vomiting & 285.9 & $195(1.9 \%)$ & Anemia, unspecified \\
\hline 284.1 & $371(1.1 \%)$ & Pancytopenia & 518.81 & $192(1.9 \%)$ & Acute respiratory failure \\
\hline 577.0 & $369(1.1 \%)$ & Acute pancreatitis & 996.85 & $188(1.9 \%)$ & Complications of transplanted bone marrow \\
\hline
\end{tabular}

\& Includes non-infectious ICD-9 codes from CCS diagnostic group 237

Ancludes 760 individual ICD-9 diagnostic codes.

\#Includes 547 individual ICD-9 diagnostic codes. 


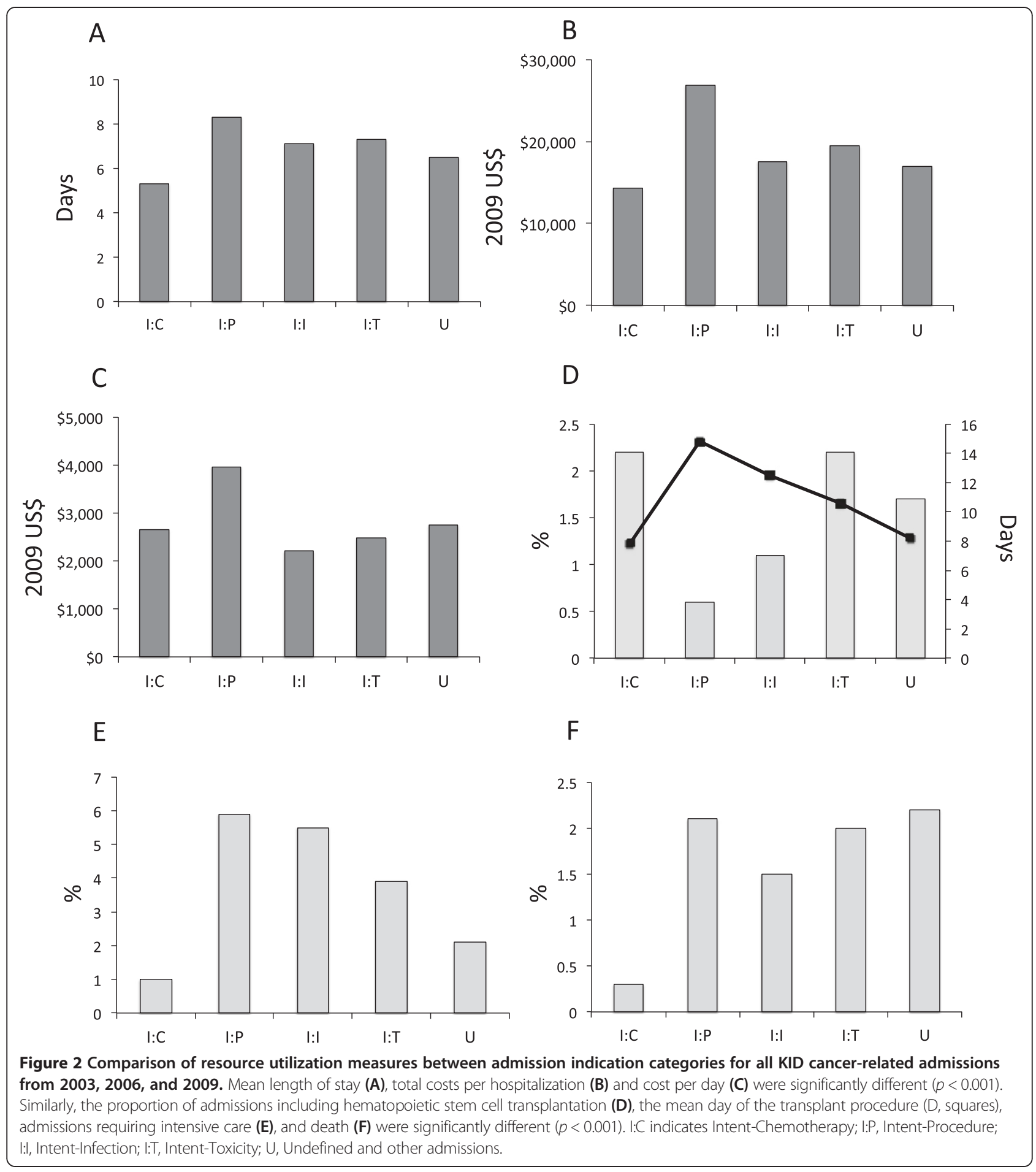

However, on average, HCT occurred earlier in IntentChemotherapy (Day 7.8) compared with Intent-Toxicity admissions (Day 10.6).

\section{Discussion}

This report describes a method of stratifying childhood cancer admissions into clinically meaningful reasons for admission. We used the externally developed CCS classification scheme based on ICD-9, which includes a combination of diagnostic and procedure codes, and used the secondary diagnostic codes when primary diagnostic codes were inadequate. We validated the use of the CCS classification scheme in our algorithm against the ICD-9 codes, and compared resource utilization between the 
admission indications. To our knowledge this is the first report to consider childhood cancer admissions in this framework.

HCUP estimated that 94,600 cancer-related admissions in 2009 cost approximately $\$ 1.9$ billion [2], a substantial proportion of US pediatric healthcare expenditure. Furthermore, the healthcare resource needs of each cancer diagnosis grew at appreciably different rates during the 2000s [2,3]. Translating such findings into strategies to improve care delivery has been slow because of a paucity of studies of patterns of inpatient care in complex diseases. Our method provides a framework for analyzing administrative data in a clinically meaningful manner.

Our method for defining a cancer-related admission cohort from the entire KID admission dataset was similar to that presented in HCUP's 2012 report on the same topic [2], with the exception of CCS groups 44 and 45 . We excluded CCS group 44 ("Neoplasms of unspecified nature or uncertain behavior") because of the predominance of rare and benign neoplasms included in this CCS group. CCS 45 ("Maintenance chemotherapy") was also excluded from the definition of our cancer cohort to limit the inclusion of a small subset of patients receiving chemotherapy for non-malignant disorders. We chose not to assess the sensitivity or negative predictive value of our approach in the 9.3 million non-cancer-related admissions because the sheer number of admissions was so large that both values were expected to be high.

The CCS categorization is designed and managed by HCUP and included in their data sets. This method has the distinct benefits of identifying cohorts of similar ICD-9 codes and adjustments for year to year code changes [22]. CCS groups are already included in the HCUP datasets, but the categorization software is available from HCUP and applicable to other platforms, enhancing the generalizability of this algorithm. In most scenarios of our analysis, the CCS groups adequately captured our desired diagnoses and procedures. However, there were scenarios, such as CCS diagnosis group 237, where the ICD-9 codes were distributed between categories. In the case of CCS group 237 we divided the group for better predictive value, but in other scenarios we allowed a loss of sensitivity because there were so few admissions with each ICD-9 code.

Our framework presupposes that the timing of procedures and discharge diagnoses can predict a general reason for admission, and, furthermore, that care would be more similar within each indication than between indications. Such suppositions are supported by the differences in overall utilization measures. Further, the lower rates of deaths and ICU services in admissions intended to deliver chemotherapy compared with other indications likely reflect a healthier patient population, i.e., admitted for pre-planned treatment after meeting health requirements rather than admitted in an emergency. $\mathrm{HCT}$ procedures, which require advanced planning and a relatively good state of patient health, occurred more frequently and earlier in the course of Intent-Chemotherapy admissions as compared with other admission indications. While more analysis is needed to understand the variation within each admission indication, the significant variation between admission indications supports our categorization of admissions.

The algorithm provides a framework for the majority of admissions. However, there remain approximately 13,000 admissions (6.4\%) with either malignancy or chemotherapy toxicity as a primary discharge diagnosis that do not fit into the framework. In addition, cytopenias without a more explanatory secondary diagnosis constituted a large proportion of the Intent-Toxicity admissions. Future analyses should consider if these admissions represent an indication subset that our framework failed to capture or if they are a product of the limitations of using discharge codes $[19,21,24,30]$. The lack of procedure dates by several states participating in KID suggests that we may have under-identified the admissions for procedures. We were able to adjust our definition of chemotherapy because of a limited number of codes, but such adjustment for over 1,000 procedure codes would be impractical and imprecise. Although some admissionsremained undefined after application of our algorithm, the admission indication categories translate to clinical practice with greater ease than grouping admissions solely by the primary diagnosis.

Patients within KID are de-identified, a structural aspect of the database which limits identification of other potentially high utilization events such as de novo diagnosis of cancer [13]. Because we could not link de-identified admission data with patients' medical records or other primary sources, external validation [30] could not be performed. Furthermore, de-identification prevented the examination of multiple admissions for the same patient; therefore, no comparison between the demographics of our cancerrelated admissions and an external source such as the SEER cancer registry $[1,13]$ was possible. KID provides the distinct advantage of a large, diverse geographic database with established methods for estimating national utilization [31], benefits which outweigh these aforementioned limitations within the context of our goal of studying patterns and trends in cancer-related inpatient resource utilization at a national level.

\section{Conclusions}

In summary, our multi-step algorithm for categorizing childhood cancer-related admissions identifies admissions with distinct patterns of resource utilization. Future studies could use this algorithm to compare trends in indications for hospitalizations between childhood cancer 
diagnoses or to allow researchers to identify subsets of similar admissions for closer examination. This framework has potential for application to childhood cancer in other administrative data sets. Although this algorithm was developed for childhood cancer, similar algorithms could be useful for grouping admissions for adult malignancies or other complex conditions.

\section{Additional files}

Additional file 1: Table S1. Clinical Classification Software (CCS) Groups Included in Diagnosis and Procedure Categories. Table identifying which CCS groups are included in each cancer-related diagnosis and procedure category.

Additional file 2: Algorithm. STATA do-file to categorize cancer-related admissions by CCS groups and sort them into mutually exclusive indications for admission.

\section{Abbreviations}

CCS: Clinical classification software; Cl: Confidence interval;

HCT: Hematopoietic stem cell transplant; HCUP: Healthcare Cost and Utilization Project; ICD-9: International statistical classification of diseases and related health problems, 9th version; ICU: Intensive care unit; KID: Kids Inpatient Database; LOS: Length of stay; PPV: Positive predictive value.

\section{Competing interests}

The authors declare that they have no competing interests.

\section{Authors' contributions}

HR conceived the study, participated in its design, data acquisition and analysis, and drafted the manuscript. VH participated in the design of the study, acquisition of data and drafting of the manuscript. FO, KK, MS, EK, JMS, WC, XD, and LF participated in study design, data analysis and drafting of the manuscript. All authors approved the final manuscript.

\section{Acknowledgements}

HR was supported by the UTHealth Innovation for Cancer Prevention Research Post-doctoral Fellowship, Cancer Prevention and Research Institute of Texas grant \# RP101503. The content is solely the responsibility of the authors and does not necessarily represent the official views of the Cancer Prevention and Research Institute of Texas. This research was performed as partial fulfillment of HR's PhD dissertation, Childhood Cancer Inpatient Utilization: Patterns, Costs, Associated Factors, and Time Trends from 2003 to 2009, which was publicly defended at the University of Texas School of Public Health, Houston, Texas, on May 3, 2013.

\section{Author details}

${ }^{1}$ Division of Management, Policy and Community Health, University of Texas School of Public Health, RAS E933, P.O. Box 20186, Houston, TX 77025, USA. ${ }^{2}$ Texas Children's Cancer and Hematology Centers, Baylor College of Medicine, 6701 Fannin, CCC 1580.00, Houston, TX 77030, USA. ${ }^{3}$ Pediatric Surgery, Baylor College of Medicine, 6701 Fannin, CCC Building 1210.00, Houston, TX 77030, USA. ${ }^{4}$ University of Texas School of Medicine, Center for Clinical Research and Evidence-Based Medicine, Houston, TX, USA. ${ }^{5}$ Division of Biostatistics, University of Texas School of Public Health, RAS E827, P.O. Box 20186, Houston, TX 77025, USA. ${ }^{6}$ Division of Epidemiology, Human Genetics and Environmental Sciences, University of Texas School of Public Health, RAS E631, P.O. Box 20186, Houston, TX 77025, USA. 'Baker Institute for Public Policy, Rice University, MS 40, P.O. Box 1892, Houston, TX 77251-1892, USA.

Received: 21 February 2014 Accepted: 29 September 2014 Published: 1 October 2014

\section{References}

1. Surveillance Epidemiology and End Results Program: Childhood Cancer by Site Incidence, Survival and Mortality. In SEER Cancer Statistics Review
1975-2010. Edited by Howlader N, Noone AM, Krapcho M, Garshell J, Neyman N, Altekruse SF, Kosary CL, Yu M, Ruhl J, Tatalovich Z, Cho H, Mariotto A, Lewis DR, Chen HS, Feuer EJ, Cronin KA. Bethesda, MD: National Cancer Institute; 2012.

2. Price A, Stranges E, Elixhauser A: Pediatric Cancer Hospitalizations, 2009. In HCUP Statistical Brief \#132. Rockville, MD: Agency for Healthcare Research and Quality; 2012.

3. Berry JG, Hall M, Hall DE, Kuo DZ, Cohen E, Agrawal R, Mandl KD, Clifton $H$, Neff J: Inpatient growth and resource use in 28 children's hospitals: a longitudinal, multi-institutional study. JAMA Pediatr 2013, 167(2):170-177.

4. Krzyzanowska MK, Treacy J, Maloney B, Lavino A, Jacobson JO: Development of a patient registry to evaluate hospital admissions related to chemotherapy toxicity in a community cancer center. $J$ Oncol Pract 2005, 1(1):15-19.

5. McKenzie H, Hayes L, White K, Cox K, Fethney J, Boughton M, Dunn J: Chemotherapy outpatients' unplanned presentations to hospital: a retrospective study. Support Care Cancer 2011, 19(7):963-969.

6. Miranda V, Fede A, Nobuo M, Ayres V, Giglio A, Miranda M, Riechelmann RP: Adverse drug reactions and drug interactions as causes of hospital admission in oncology. J Pain Symptom Manage 2011, 42(3):342-353.

7. Baena-Canada JM, Estalella-Mendoza S, Rosado-Varela P, Exposito-Alvarez I, Gonzalez-Guerrero M, Diaz-Blanco MC, Cortes-Carmona C, Ramirez-Daffos P, Arriola-Arellano E, Rueda-Ramos A, Solana-Grimaldi L, Benitez-Rodriquez E: Use of health-care services during chemotherapy for breast cancer. Eur J Cancer 2012, 48(18):3328-3334.

8. Sung L, Aplenc R, Alonzo TA, Gerbing RB, Lehrnbecher T, Gamis AS: Effectiveness of supportive care measures to reduce infections in pediatric AML: a report from the Children's Oncology Group. Blood 2013, 121(18):3573-3577.

9. Werba BE, Hobbie W, Kazak AE, Ittenbach RF, Reilly AF, Meadows AT: Classifying the intensity of pediatric cancer treatment protocols: the intensity of treatment rating scale 2.0 (ITR-2). Pediatr Blood Cancer 2007, 48(7):673-677.

10. Maeda JL, Raetzman SO, Friedman BS: What hospital inpatient services contributed the most to the 2001-2006 growth in cost per case. Health Serv Res 2012, 47(5):1814-1835.

11. Sun Y, Friedman B: Tools for more accurate inpatient cost estimates with HCUP databases, 2009. Rockville, MD: Agency for Healthcare Research and Quality; 2011.

12. Lopez AM, Tilford JM, Anand KJS, Jo C-H, Green JW, Aitken ME, Fiser DH: Variation in pediatric intensive care therapies and outcomes by race, gender, and insurance status. Pediatr Crit Care Med 2006, 7(1):2-6.

13. Fisher BT, Harris T, Torp K, Seif AE, Shah A, Huang YS, Bailey LC, Kersun LS, Reilly AF, Rheingold SR, Walker D, Li Y, Aplenc R: Establishment of an 11-year cohort of 8733 pediatric patients hospitalized at United States free-standing children's hospitals with de novo acute lymphoblastic leukemia from health care administrative data. Med Care 2014, 52(1):e1-e6.

14. Kavcic M, Fisher BT, Li Y, Seif AE, Torp K, Walker DM, Huang YS, Lee GE, Tasian SK, Vujkovic M, Bagatell R, Aplenc R: Induction mortality and resource utilization in children treated for acute myeloid leukemia at free-standing pediatric hospitals in the United States. Cancer 2013, 119(10):1916-1923.

15. Shaw PH, Ritchey AK: Different rates of clinical trial enrollment between adolescents and young adults aged 15 to 22 years and children under 15 years old with cancer at a children's hospital. $J$ Pediatr Hematol Oncol 2007, 29(2):811-814.

16. Koschmann C, Thomson B, Hawkins DS: No evidence of a trial effect in newly diagnosed pediatric acute lymphoblastic leukemia. Arch Pediatr Adolesc Med 2010, 164(3):214-217.

17. Hunger SP, Lu X, Devidas M, Camitta BM, Gaynon PS, Winick NJ, Reaman GH, Carroll WL: Improved survival for children and adolescents with acute lymphoblastic leukemia between 1990 and 2005: a report from the children's oncology group. J Clin Oncol 2012, 30(14):1663-1669.

18. Hendrickson KC, Rimar J: Patterns of hospital resource utilization of children with leukemia and CNS tumors: a comparison of children who survive and those who die within 3 years of diagnosis. Nurs Econ 2009, 27(1):35-44

19. Cooke CR, Iwashyna TJ: Using existing data to address important clinical questions in critical care. Crit Care Med 2013, 41(3):886-896.

20. Healthcare Costs and Utilization Project: Introduction to the HCUP KIDS' inpatient database. Rockville, MD: HCUP; 2011. 
21. Woodworth GF, Baird CJ, Garces-Ambrossi G, Tonascia J, Tamargo RJ: Inaccuracy of the administrative database: comparative analysis of two databases for the diagnosis and treatment of intracranial aneurysms. Neurosurgery 2009, 65(2):251-256. discussion 256-257.

22. Elixhauser A, Steiner C, Palmer L: Clinical Classifications Software (CCS), 2004. Rockville, MD: Agency for Healthcare Research and Quality; 2004.

23. Berry SA, Fleishman JA, Moore RD, Gebo KA: Trends in reasons for hospitalization in a multisite United States cohort of persons living with HIV, 2001-2008. JAIDS 2012, 59(4):368-375.

24. Centers for Medicare and Medicaid Services and the National Center for Health Statistics: ICD-9-CM Official Guildlines for Coding and Reporting, Effective October 1, 2008. 2008.

25. Newcombe RG: Two-sided confidence intervals for the single proportion: comparison of seven methods. Stat Med 1998, 17:857-872.

26. Lin YF, Lairson DR, Chan W, Du XL, Leung KS, Kennedy-Nasser AA, Martinez CA, Gottschalk SM, Bollard CM, Heslop HE, Brenner MK, Krance RA: The costs and cost-effectiveness of allogeneic peripheral blood stem cell transplantation versus bone marrow transplantation in pediatric patients with acute leukemia. Biol Blood Marrow Transplant 2010, 16(9):1272-1281

27. Russell HV, Panchal J, Vonville H, Franzini L, Swint JM: Economic evaluation of pediatric cancer treatment: a systematic literature review. Pediatrics 2013, 131(1):e273-e287.

28. U.S. Agency for Healthcare Research and Quality: Cost-to-Charge Ratio Files: 2006 Kids' Inpatient Database (KID) User Guide. Rockville, MD: U.S. Agency for Healthcare Research and Quality; 2008.

29. Consumer Price Index. [http://data.bls.gov/cgi-bin/cpicalc.pl]

30. Benchimol El, Manuel DG, To T, Griffiths AM, Rabeneck L, Guttmann A: Development and use of reporting guidelines for assessing the quality of validation studies of health administrative data. J Clin Epidemiol 2011, 64(8):821-829.

31. Chu B, Houchens R, Elixhauser A, Ross D: Using the KIDS' Inpatient Database (KID) to Estimate Trends. Rockville, MD: Agency for Healthcare Research and Quality; 2007.

doi:10.1186/1472-6947-14-88

Cite this article as: Russell et al:: Algorithm for analysis of administrative pediatric cancer hospitalization data according to indication for admission. BMC Medical Informatics and Decision Making 2014 14:88.

\section{Submit your next manuscript to BioMed Central and take full advantage of:}

- Convenient online submission

- Thorough peer review

- No space constraints or color figure charges

- Immediate publication on acceptance

- Inclusion in PubMed, CAS, Scopus and Google Scholar

- Research which is freely available for redistribution 